SLAC-PUB-CERN-TH.7273/94

May 1994

hep-th/9405xxx

\title{
Effects of Virtual Monopoles
}

\author{
A. De Rujula \\ CERN, 1211 Geneva 23 \\ Switzerland
}

\begin{abstract}
Electromagnetism would be a "more unified" theory if there were elementary magnetic monopoles and/or particles with both electric and magnetic charges (dyons). I discuss the simplest possibilities for the addition of these entities onto the Standard Model, and their empirical consequences. Lower limits on the masses of monopoles and dyons stemming from their quantum effects on current observables turn out to be much stronger than the existing limits from direct searches. Anomalies in the three-photon decay of the $Z$ constitute good specific signatures for monopoles or dyons. T-odd observables in the $e^{+} e^{-} \rightarrow W^{+} W^{-}$process are signatures for dyons, but they are severely constrained by existing data. The subjects of monopolium, monopole cosmology and non-elementary monopoles are also discussed.
\end{abstract}

CERN-TH.7273/94

May 1994 


\section{INTRODUCTION}

The quest for symmetries in the laws of Nature, sometimes exact, occasionally broken in more or less elegant ways, has been crowned by a long list of successes. This, and not only aesthetical pleasure, is a good reason to pursue the quest. Often quoted as the first, or second, "grand unification" is that of electricity and magnetism. But Maxwell's or Nature's feat is only partial: the conceivable symmetry between electric and magnetic charges is broken by the non-existence of (relatively light) magnetic monopoles.

A higher symmetry was one of Dirac's motivations to introduce the notion of magnetic charges [1]. But he found that the elementary electric and magnetic charges $e$ and $g$ ought to be quantified so that $e g=2 \pi n$, with $n$ an integer (units are such that $\left.\alpha_{e} \equiv e^{2} / 4 \pi \sim 1 / 137, \alpha_{g} \equiv g^{2} / 4 \pi \sim 34 n^{2}\right)$. Thus, in a sense, the addition of Dirac's monopoles to QED would not constitute a truly "grand" unification of electricity and magnetism, since $e \neq g$. Perhaps this asymmetry can be cured as in conventional Grand-Unified Theories, by recalling that, in a quantum theory, couplings are energy-dependent and it is only at a scale wherein masses are negligible that the couplings merge into a single value (the Dirac condition $e\left(q^{2}\right) g\left(q^{2}\right)=2 \pi n$ has been argued to hold [2] for the one-loop renormalized quantities at any given momentum scale $q^{2}$, so that as $e$ increases with $q^{2}$, $e$ and $g$ do tend to merge).

Interest in monopoles revived in the 70's, following the discovery by 't Hooft and Polyakov [3] that there exist monopole solutions to the field equations of theories in which a semi-simple unifying gauge group is broken to the $U(1)$ of QED. If the unification scale is $M_{\mathrm{GUT}}$, the monopole mass [4] is $m \geq M_{\mathrm{GUT}} / \bar{\alpha}$, with $\bar{\alpha}$ the grand-unified fine-structure constant. A consequence of these theoretical developments is that the feeling has permeated the community that monopoles are extremely heavy objects, desperately out of the reach of accelerator experiments. I perceive this circumstance as negative and I contend that monopoles much lighter than the "grand-unified" ones may simply happen to exist, and should be looked 
for.

Almost everywhere, I assume monopoles to be spin-1/2 "point-like" particles whose radius, $R$, is negligible relative to the inverse of their mass, in contrast to grand-unified monopoles, extensive objects with $R \sim 1 /(m \bar{\alpha})$. In Section 7, I discuss monopoles that are not point-like, but are unconventional in being much lighter than the grand-unified ones.

The extension of QED to a theory containing both electric and magnetic elementary charges is non-trivial and, in the case of point-like monopoles, it has not reached the theoretically satisfactory point wherein consistency and renormalizability have been demonstrated to all orders of perturbation theory (even if one were to finesse the problem that a perturbative expansion cannot be simultaneously convergent in $e$ and $g$ ). The Feynman rules have been derived [5-8] and are consistently usable at the one-loop level, in the case of monopoles. For dyons the situation is more obscure (these points are discussed in Section 2).

For the purposes of this paper, to estimate the current limits on monopole masses and to suggest concrete strategies for indirect monopole searches, our current understanding of monopole theory is (almost) all one needs.

The standard $S U(2) \otimes U(1)_{Y}$ model is sufficiently well established for it to be necessary, when considering monopoles, to discuss the gauge-group representation to which they might belong. To avoid anomalies and other potential strictures, one is most economically led to introduce "vector-like" spin-1/2 monopoles, whose left- and right-handed projections belong to the same group representation. I consider only the two simplest cases, that of electrically neutral $S U(2)$-singlet monopoles $M_{L, R}^{0}$ and that of "dyons" $\left(D^{0}, D^{-}\right)_{L, R}$, doublets with $\left(\nu^{0}, e^{-}\right)$-like electrical charges ${ }^{\star}$. In what follows I refer to these two choices simply as monopoles and dyons, or to both as monopoles when no distinction is necessary.

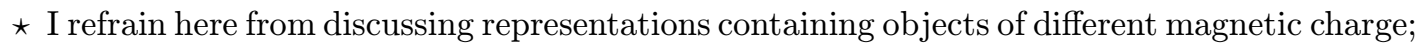
this would lead to the introduction of magnetically-charged gauge bosons and an enlarged gauge group.
} 
In both cases the magnetic charge is commensurate a la Dirac: $g=2 \pi n / e$. To economize parameters, I generally let the dyons be (quasi)-degenerate, $\mid m\left(D^{-}\right)-$ $m\left(D^{0}\right) \mid \ll m\left(D^{i}\right)$.

The current to which the standard $Z^{0}$ boson couples has an electromagnetic component, so that not only photons, but also $Z$ 's must couple to monopoles, with a large amplitude of $O(g)$. If monopoles are not confined, as they might [9] by the strong monopole-antimonopole forces, the fact that LEP detectors have not been swamped by monopoles immediately implies a "direct" limit $m>M_{z} / 2$.

We show in Section 3 how the trumpeted success of the Standard Model in accommodating all current data implies a lower limit on the monopole mass of order $1 \mathrm{TeV}$, a much stronger result than the direct one ${ }^{\dagger}$. In Section 4 we discuss how the $Z$ decays into odd numbers of photons constitute a similarly sensitive but much more specific limiting (or discovery) test. The masses of our dyons are constrained by current data in the same way as those of monopoles; in Section 6 we discuss how dyons, which couple not only to $\gamma$ 's and $Z$ 's, but also to $W$ 's, may induce very characteristic $T$-odd observables in the $e^{+} e^{-} \rightarrow W^{+} W^{-}$process. But the available data constrain these effects below the level of observability at LEP-II. I do not discuss monopole signatures at higher-energy $e^{+} e^{-}$colliders.

The monopole charge is too large for a perturbative expansion in $g$ to be trustworthy. This unsurmounted caveat will unavoidably haunt all our numerical considerations: an argument is needed to judge the credibility of the results.

Throughout the paper, we discuss how monopoles may modify the standard relations between observables measured at energy scales below the monopole mass, $q^{2}<m^{2}$. The incidence of monopoles can thus be characterized in the customary fashion, by grafting onto the standard Lagrangian a series of dimension $d>4$ effective operators induced by virtual monopoles, whose degrees of freedom have

$\dagger$ This is so in spite of the fact that, with our choice of monopole properties, the "oblique" vector-boson propagator corrections $S, T$ and $U$, at least in their original disguise [10], vanish automatically. 
been integrated out, or, more pictorially, integrated "in". In each case we characterize the effect of monopoles by the lowest-dimension relevant operator (this is tantamount to keeping the first non-vanishing term in an expansion in $q^{2} / \mathrm{m}^{2}$ ). All we assume is that the order of magnitude of the coefficient of each of these operators can be estimated by computing it to lowest non-vanishing order in $g$. This is indeed an assumption: in every case the corrections (to the next order in $g$ ) are $O\left(g^{2} / 16 \pi^{2}\right) \sim 1$.

Equivalent to the above is the approach used in other strongly-interacting realms, such as the chiral Lagrangians describing the low-energy interactions of pseudoscalar mesons. There, the size of the coefficient of a particular operator is set by demanding that its estimates to successive orders of perturbation give results of the same magnitude [11]. The coefficients of the effective operators we deal with involve powers of $g / m$. In a "chiral-like" approach one would simply substitute a multiple of $g / m$ by the analogue of $f_{\pi}^{-1}$. In a sense, computing diagrams explicitly, as we do, is simply a way to keep straight the powers of $4 \pi$.

Monopole-antimonopole bound states may occur below the threshold for "open" monopole production, and need to be discussed. I argue in Section 5 that these states are so wide that they should not be considered explicitly. Their collective effects should already be included in the "partonic" description in terms of virtual-monopole loops.

Arbitrarily heavy elementary monopoles or dyons would antagonize the standard cosmological lore. The ensuing upper mass limits are discussed in Section 8. A summary and the conclusions are offered in Section 9.

Since the subject of monopoles has not been investigated at length in the spirit of this paper, I have taken the liberty to report on the results of the exploration of various alleys which do not take to fertile destinations. Consequently, a positivethinking reader may consider skipping Sections 5 to 7, which deal with monopolia, $W$-pair production and extended monopoles, and to concentrate on Section 4 , that deals with the phenomenologically relevant subject of the multiphoton decays of 
the $Z$.

\section{EleCtrodynamics With ElECtric AND MAGNetic POLES}

It is not possible to write a theory of point-like electric and magnetic charges wherein the electromagnetic field is described exclusively by a local vector potential $A^{\mu}(x)$. Whence the necessity of introducing the Dirac string [1] or a multivalued potential [12]. The string, or its surrogates, can be regarded as gauge-artefacts and are unobservable if the Dirac charge-quantization condition is satisfied. Yet, they entail a measure of non-locality in the action and in probability amplitudes, that must not make its way to the observables.

An action describing electric and magnetic poles can be translated with the customary methods into a set of Feynman rules. Very little attention has been paid to these rules, the conventional wisdom (that I have challenged in the Introduction) being that the large value of $g$ makes them entirely useless. These calculational rules are of delicate handling in the case of monopoles, and are worse than delicate for dyons.

Let $n$ be a space-like four-vector tangent to the Dirac string, $\epsilon_{\mu \nu \rho \sigma}$ the fully antisymmetric four-index symbol, and $\epsilon_{A}^{\rho}$ a photon-polarization vector. The monopole counterparts to the $\mathcal{L}_{e}=-i e A \cdot J$ coupling of a photon to a charged object, the configuration- and momentum-space vertex "Feynman rules" for the coupling to a monopole, are [6-8]:

$$
\mathcal{L}_{g}=-i g \frac{\epsilon\left(n, \partial_{x}, A(x), J(x)\right)}{n \cdot \partial_{x}} \leftrightarrow-i g \frac{\epsilon_{\mu \nu \rho \sigma} n^{\mu} q^{\nu} \epsilon_{A}^{\rho} J^{\sigma}}{n \cdot q+i \epsilon}
$$

with $J_{\sigma}=\left(p_{\sigma}+p_{\sigma}^{\prime}\right)$ or $\bar{\psi}\left(p^{\prime}\right) \gamma_{\sigma} \psi(p)$, for spinless and spin-1/2 monopoles. The $\epsilon$ tensor is needed to describe a "dual" coupling, and the vector $n$ must be contracted with it, since there are only two independent four-vectors in the set $q, p, p^{\prime}$. The terrifying denominators in Eqs. (1) embody the non-local character of the Dirac string. 
Our $M_{L, R}^{0}$ monopoles and $\left(D^{0}, D^{-}\right)_{L, R}$ dyons are "magnetic" weak-SU(2) singlets, but they carry $U(1)_{Y}$ magnetic charge. Their couplings to $Z$ 's and $\gamma$ 's are the dual or magnetic counterparts to the standard couplings of a singlet with nonvanishing hypercharge:

$$
\mathcal{L}_{D}=-i\left(\frac{g}{c}\right) \frac{\epsilon\left(n, \partial_{x}, B(x), J(x)\right)}{n \cdot \partial_{x}}
$$

where

$$
B_{\mu} \equiv c A_{\mu}-s Z_{\mu}
$$

is the $U(1)_{Y}$ potential, and $s, c=\sin \left(\theta_{W}\right), \cos \left(\theta_{W}\right)$. Our electrically-neutral monopoles, $M^{0}$, have no gauge couplings other than the ones of Eq. (2), while dyons also sport standard "electric" vector-like couplings to $W$ 's, to $Z$ 's and, in the case of $D^{-}$, to $\gamma^{\prime}$ s.

To illustrate the use of Eq. (1), consider the photon-mediated processes of $e^{+} e^{-}$ annihilation into lepton $(L)$, monopole $(M)$ or dyon $(D)$ pairs. The matrix element squared for $L^{+} L^{-}$production is of the form $\left|J_{e}^{\dagger} \cdot J_{L}\right|^{2} / q^{4}$. The calculation of the cross section for $e^{+} e^{-} \rightarrow M \bar{M}$ production with casual use of the rule (1) leads to an $n$-dependent result, even after the erasure of all terms that vanish because of current conservation $\left(q \cdot J_{e}=q \cdot J_{M}=0\right)$. The somewhat debonair recipe [8] of dropping all terms proportional to $q^{2}$ (that would cancel the photon's propagator pole) results in a squared matrix element proportional to $\left(\left|J_{e}^{\dagger} \cdot J_{M}\right|^{2}-\left|J_{e}\right|^{2}\left|J_{M}\right|^{2}\right) / q^{4}$, a sensible answer ${ }^{\star}$ at last. The total cross sections for the production of magnetically and electrically charged pairs of a given spin have the same functional form, as expected; while their angular distributions are different, also as expected.

So far, so good. The troubles arise as one attempts to extend these considerations to the $e^{+} e^{-}$production of dyon pairs. The Feynman rules are simply

* The crossed process of electron scattering on a monopole has the same squared matrix element, from which one can reproduce the classical results for the scattering on a static monopole of electrons that do not hit the string. 
obtained [7] by adding to Eq. (1) a conventional electric-charge coupling of the $D^{-}$ dyon. The interference term between the "electric" and "magnetic" dyon amplitudes does not cancel in the expression for the cross section and is proportional to $\epsilon\left(n, p, p^{\prime}, k\right)$ with $k$ one of the incoming momenta and $n$, alas, the vector defining the Dirac string. I have neither solved this problem nor found it discussed in the literature. Clearly, the bugaboo is in the treatment of superimposed singularities at "the end of the string". Presumably a study of the effective vertex operators describing the long-wavelength limit of the photon couplings to (not point-like) topological dyons would help clarify these issues.

The slippery character of all these grounds emanates from the necessity of using vector potentials, rather than $\vec{E}$ and $\vec{B}$ fields, in describing photonic couplings to monopolar electric and magnetic charges. There is no difficulty in translating a magnetic-dipole coupling such as $F_{\mu \nu} \bar{\psi} \sigma^{\mu \nu} \psi$ into its dual electric-dipole counterpart, suffice it to trade $F_{\mu \nu}$ for $\tilde{F}_{\mu \nu} \equiv \frac{1}{2} \epsilon_{\mu \nu \rho \sigma} F^{\mu \nu}$. This simple remark will allow us, in discusssing dyons in Section 6, to bypass the problem of dealing with inconsistent Feynman rules.

\section{Current limits on the masses of monopoles AND Dyons}

It has become a flourishing industry to set limits on novel effects by exploiting the accurate success of the Standard Model in relating precise electroweak data at energies up to $M_{z}$ (see [13] for a clear review). Typically, three of the best measured quantities $\left(\alpha, M_{z}\right.$ and the Fermi constant as extracted from muon beta decay) are used as inputs to specify the parameters and predict other quantities. The predictions depend significantly on the mass of the top quark, $m_{t}$, and to a lesser extent on the Higgs boson mass, $M_{H}$.

The CDF group has announced [14] evidence for the $t$ quark, at $m_{t}=175 \pm 15$ $\mathrm{GeV}$, a result that we take into account where appropriate. The uncertainty in $M_{H}$ can be conveniently dealt with by allowing it to vary from its experimental lower limit $(\sim 50 \mathrm{GeV})$ to some theoretically sensible upper limit $(\sim 1 \mathrm{TeV})$ 
and adding the effect linearly as a "theoretical error" in the prediction at hand. Comparison with measured values of the predicted quantities then results in limits on the parameters of some non-standard effect, as functions of $m_{t}$. In dealing with limits on the mass of monopoles, we follow this traditional procedure.

The most relevant predicted observables in constraining monopole masses turn out to be $g_{A}$ and $g_{V} / g_{A}$, the vector and axial couplings of the $Z$ to charged leptons (extracted from the $Z$ leptonic widths and asymmetries) and $M_{W} / M_{Z}$. For ease of reference, I collect below the standard results (the data and analysis are as in [13], and the $m_{t}$-dependences are my own simplistic fits to the full predictions of the Standard Model, sufficiently accurate over the relevant $m_{t}$ range; the $1 \sigma$ errors in the predictions are given in parenthesis, the theoretical Higgs-related error is labelled $\left.\left[M_{H}\right]\right)$ :

$$
\begin{gathered}
\frac{g_{V}}{g_{A}}=0.0753(12)+0.00345\left[\frac{m_{t}^{2}-(176 \mathrm{GeV})^{2}}{M_{Z}^{2}} \pm 1.0\left[M_{H}\right]\right], \\
-g_{A}=0.5(0)+0.00065\left[\frac{m_{t}^{2}-(111 \mathrm{GeV})^{2}}{M_{Z}^{2}} \pm 0.4\left[M_{H}\right]\right], \\
\frac{M_{W}}{M_{Z}}=0.8768(2)+0.00163\left[4.25 \frac{m_{t}-100 \mathrm{GeV}}{M_{Z}} \pm 0.6\left[M_{H}\right]\right] .
\end{gathered}
$$

These are to be compared with the experimental results:

$$
\frac{g_{V}}{g_{A}}=0.0728(28) \quad-g_{A}=0.4999(9) \quad \frac{M_{W}}{M_{Z}}=0.8798(29)
$$

Let $V=\{\gamma, Z\}$ represent the pair of neutral gauge bosons. Virtual monopoles contribute to the $V$-propagator via diagrams such as those in Fig. 1, and modify the standard predictions for the observables mentioned in the previous paragraph. As discussed in the Introduction, we estimate the effect of monopoles by focusing on the pertinent lowest-dimension effective operator (induced by integrating "in" 
the monopole degrees of freedom) and computing its coefficient to leading order in $g^{2}$. In the case of the $V$-propagator, this is equivalent to computing, to $O\left(\mathrm{~m}^{-2}\right)$, the $O\left(g^{2}\right)$ diagram of Fig. 1a and neglecting corrections of $O\left(g^{2} / 16 \pi^{2}\right)$ or higher, such as those represented by the diagram of Fig. $1 \mathrm{~b}$.

The results for the putative departures $(\operatorname{denoted} \Delta)$ from the standard predictions, are:

$$
\begin{gathered}
\Delta \frac{g_{V}}{g_{A}}=\frac{16 s^{4} c^{2}}{c^{2}-s^{2}} \frac{g^{2}}{4 \pi^{2}} \frac{M_{Z}^{2}}{15 m^{2}}, \\
\Delta\left(g_{A}\right)=\frac{s^{2}}{c^{2}} \frac{g^{2}}{4 \pi^{2}} \frac{M_{Z}^{2}}{15 m^{2}} \\
\Delta \frac{M_{W}}{M_{Z}}=\frac{c s^{2}}{c^{2}-s^{2}} \frac{g^{2}}{4 \pi^{2}} \frac{M_{Z}^{2}}{15 m^{2}},
\end{gathered}
$$

where $s^{2} \equiv 1-c^{2} \equiv \sin ^{2}\left(\theta_{W}\right) \sim 0.231^{\star}$.

The various $1 \sigma$ and $3 \sigma$ limits on the monopole mass $m$ as functions of $m_{t}$ are respectively displayed in Figs. $2 \mathrm{a}$ and $2 \mathrm{~b}$, for a monopole of minimum magnetic charge, $n=1$ (since the monopole-induced corrections " $\Delta$ " are all quadratic in $g / m$, the figure can also be interpreted as the limits on $m / n$, for a monopole of charge $g=2 \pi n / e$, with $n \geq 1$ ). The combined lower limits are:

$$
\begin{aligned}
& m>n \times 1.0 \mathrm{TeV}, \text { at } m_{t}=147 \mathrm{GeV}(1 \sigma), \\
& m>n \times 0.7 \mathrm{TeV}, \text { at } m_{t}=137 \mathrm{GeV}(3 \sigma),
\end{aligned}
$$

where in the numerics I have used the value of $g$ that corresponds to $\alpha_{e}\left(M_{z}^{2}\right) \sim$ 1/128. As can be seen in Fig. 2, the recent CDF result [14] on $m_{t}$ modifies Eq. (11)

$\star$ Notice that with our conventions for $e$ and $g$ and the corresponding currents, these corrections are a factor of two larger than the ones that would be obtained by first computing the effect of a singlet vector-like heavy lepton $E^{-}$of mass $m$ and then substituting $e$ for $g$ in the result. 
to

$$
m>n \times 1.2 \mathrm{TeV}, \text { at } m_{t}=165 \mathrm{GeV}(1 \sigma),
$$

while the $3 \sigma$ result of Eq. (12) stays put.

For our mass-degenerate dyons, the $D^{0}$ and the $D^{-}$loop give contributions to the various $\Delta$ 's identical to those of a singlet monopole and the above lower-mass

limits are to be multiplied by $\sqrt{2}$. At first sight, the dealer in the art of setting limits on physics beyond the Standard Model may be surprised that, in spite of their strong couplings, monopoles and dyons are not more constrained than in Eqs. (11)-(13). The reason for the relative weakness of these strictures is that vector-like monopoles and quasi-degenerate dyons are very particularly guileful at avoiding all low-energy constraints.

Given our perilous use of a perturbative expansion in $g$, Eqs. (11)-(13) are only estimates of monopole-mass limits. But these limits are so much larger than $M_{Z}$ that it is fair to conclude that direct searches for monopoles at LEP are unlikely to be successful ${ }^{\dagger}$.

\section{Z DECAYS INTO MULTIPLE PHOTONS}

There are ways to search for virtual-monopole effects more specific than the ones discussed in the previous Section. Given the very large couplings of monopoles to $Z$ 's and $\gamma$ 's, the most obvious candidate is the monopole-mediated decay of a $Z$ into an odd number of photons, $l$, as depicted in Fig. 3a for $l=3$. Because of the naturally large couplings of monopoles to the gauge bosons, it is difficult to imagine a sensible theory that, for a fixed scale of its new dynamics, could modify $Z \rightarrow l \gamma$ decays as much as monopoles would.

Even for the dominant $(l=3)$ decay, the standard $Z \rightarrow l \gamma$ transition, induced by all real and virtual charged-particle intermediate states, has a totally negligible

$\dagger$ To a lesser extent, this grim conclusion could also have been reached from a previous analysis, along similar lines, of the monopole contributions to the anomalous magnetic moment of the muon [15], that result in a limit $m>120 \mathrm{GeV}$. 
branching ratio $\sim 2.8 \times 10^{-10}[17]$. The main conventional source of $3 \gamma$ final states at $\sqrt{s}=M_{z}$ is the pure QED $e^{+} e^{-}$annihilation depicted in Fig. 3d. This process has a characteristic photon-bremsstrahlung behaviour, with its cross section peaking when one of the photons is soft, or collinear to one of the colliding particles. For photons sufficiently hard an acollinear to be observable in LEP detectors, $\sigma\left(e^{+} e^{-} \rightarrow 3 \gamma\right) / \sigma_{\text {TOT }} \sim$ a few $10^{-6}$ at $\sqrt{s}=M_{z}$ [18]. This means that for millions of produced $Z$ 's, the QED process is marginally observable ${ }^{\ddagger}$.

Since the standard $e^{+} e^{-} \rightarrow 3 \gamma$ process is not negligible, in studying a possible deviation from the expectations one ought to compute the interference of the QED amplitude of Fig. 3d with that for a monopole-induced transition, Fig. 3a. Prior to the discovery of a significant excess of $3 \gamma$ final states (or of a departure from the QED-predicted angular and energy distributions), it appears reasonable to refrain from this laborious task. For our current purposes, it suffices to deal with the pure monopole-induced $Z \rightarrow l \gamma$ partial widths.

We estimate the virtual-monopole mediated $Z \rightarrow l \gamma$ amplitudes by computing to leading order in $g$ the corresponding lowest-dimension effective operators. This involves a calculation of Feynman diagrams such as that of Fig. 3a, and the neglect of higher-order effects like the one illustrated by Fig. 3c. In the figure we emphasize the distinction between the conventional $Z-e$ vertex and the dual $\{Z, \gamma\}$-monopole couplings of Eqs. (2),(3) by denoting the latter with a dot. The simplest way to present the results is to trade the calculation of Fig. 3a for that of Fig. 3b, that is, to use the theory's duality to treat the electron as a monopole and the monopole as a conventional QED charged lepton, while keeping straight the powers of $e$ and $g$ and substituting $\bar{\psi}_{e} \gamma_{\sigma}\left(g_{V}+g_{A} \gamma_{5}\right) \psi_{e}$ for $J_{\sigma}$ in Eq. (1).

But for a rescaling of coupling constants, we have reduced our task to the calculation of the effective interactions describing the couplings of an even number $(l+1)$ of vector bosons, induced at the one-loop level by a massive charged spinor

$\ddagger$ There are clearly other detector-related backgrounds, such as $\pi^{0}$ 's or even jets masquerading as single photons, but these are not for a theorist to deal with. 
field to which they couple as in QED. This is precisely the definition of the EulerHeisenberg Lagrangian [16] describing light-by-light scattering (and its generalizations) at scales below $m_{e}$. The effective interactions relevant to monopole-induced $Z \rightarrow 3 \gamma$ and $Z \rightarrow 5 \gamma$ transitions are:

$$
\begin{gathered}
\mathcal{L}_{3}=\frac{\alpha_{g}^{2}}{360 m^{4}} \frac{s}{c}\left\{4(F \cdot F)^{2}+7(F \cdot \widetilde{F})^{2}\right\}, \\
\mathcal{L}_{5}=\frac{\pi \alpha_{g}^{3}}{22680 m^{8}} \frac{s}{c}\left\{8(F \cdot F)^{3}+13(F \cdot F)(F \cdot \widetilde{F})^{2}\right\},
\end{gathered}
$$

where $F \cdot F=F^{\mu \nu} F_{\mu \nu}$, to be properly symmetrized over all photons. Notice the factor $s / c=\tan \theta_{W}$ arising from the substitution of one photon by a vectoriallycoupled $Z$ in the conventional multiphoton effective Lagrangian. Given these results, it is only a matter of some toil to compute the rates, energy- and angulardistributions of the final-state photons.

It is convenient to discuss first the total cross sections $\sigma\left(e^{+} e^{-} \rightarrow Z \rightarrow l \gamma\right)$, and to re-express them as $Z$ partial widths. For $l=3$ :

$$
\Gamma(Z \rightarrow 3 \gamma)=M_{Z} \frac{17 \pi s^{2}}{972000 c^{2}}\left[\frac{g^{2}}{4 \pi^{2}}\right]^{4}\left(\frac{M_{Z}}{m}\right)^{8}
$$

while, for $l=5^{\star}$ :

$$
\Gamma(Z \rightarrow 5 \gamma)=M_{Z} \frac{\pi s^{2}}{2^{25} 3^{11} 5^{2} 7^{2} c^{2}}\left[\frac{g^{2}}{4 \pi^{2}}\right]^{6}\left(\frac{M_{Z}}{m}\right)^{16}\left\{3.92 \times 10^{-4}\right\}
$$

Notice that the r.h.s. of Eq. (16) is a function of $m / n$, with $n$ the monopole charge in elementary units, and recall that the other monopole-induced effects of

$\star$ Here an overall factor $1 /\left(2^{30} 3^{3} \pi^{7}\right)$ is phase space, the result for an $\mathcal{L}_{5}=\phi^{6} / M_{z}^{2}$ coupling between distinguishable scalars. The quantity in curly brackets is an estimate (of better than $1 \%$ precision) of the average over the eight-dimensional phase space of the normalized matrix element squared, i.e. the result of substituting the six $F$ 's within the curly brackets of Eq. (15) by all permutations of $F_{1} \ldots F_{6}$, adding, dividing by $6 ! M_{z}^{6}$, squaring, and summing over polarizations; the smallness of this number reflects the derivative nature of the coupling to the five photons that share the available energy. 
Eqs. (8)-(10) also depend on $m / n$. Thus, in a search for virtual-monopole signatures, the relative merits of the indirect limits of Section 3 and of the more specific $Z \rightarrow 3 \gamma$ rate are independent of $n$.

Figure 4 shows Eq. (16) converted into a branching ratio with use of $\Gamma_{z}^{\text {Tот }}=$ $2.487 \mathrm{GeV}$, and plotted as a function of $m / n$. An anomaly in this branching ratio of $O\left(10^{-6}\right)$ is currently observable at LEP-I, and corresponds to $m=n \times 0.60$ $\mathrm{TeV}$. This mass value is close to the limit of Eq. (12) and, given the uncertainties inherent in a perturbative expansion in $g$, it is fair to conclude that the search for anomalies in $Z \rightarrow 3 \gamma$ transitions should be pursued with vigour. Consequently, I give more details on these decays.

Let $x_{i} \equiv 2 E_{i} / M_{Z}$ be the thrust of the individual photons, with $0 \leq x_{i} \leq 1$ and $\sum_{i} x_{i}=2$. The Dalitz plot density is:

$$
\frac{d \Gamma}{\Gamma d x_{1} d x_{2} d x_{3}}=\frac{5}{34}\left[139 \sum_{i} x_{i}^{2}\left(1-x_{i}\right)^{2}-18 \prod_{i}\left(1-x_{i}\right)\right] \delta\left(\sum_{i} x_{i}-2\right) .
$$

The single-photon energy distribution is:

$$
\frac{1}{\Gamma} \frac{d \Gamma}{d x}=\frac{x^{3}\left(3430-6210 x+2919 x^{2}\right)}{102} .
$$

The photons' angular distribution relative to the beam axis (three entries per event) is:

$$
\frac{d \sigma}{d \cos \theta} \propto 1-\frac{77}{479} \cos ^{2}(\theta)
$$

The angular distribution of the most energetic photon relative to the beam direction is:

$$
\frac{d \sigma}{d \cos \Theta} \propto 1-0.1762 \cos ^{2}(\Theta)
$$

The distribution in the angle between the normal to the three-photon plane and 
the beam axis is:

$$
\frac{d \sigma}{d \cos \alpha} \propto 1+\frac{163}{671} \cos ^{2}(\alpha)
$$

All of these angular distributions are fairly uniform.

For a monopolar mass above the limits discussed in Section 3, the ratio of fiveto three-photon decay widths of the $Z$, as obtained from Eqs. (16),(17) is a tiny number; the enhancement due to the extra powers of $g$ is compensated by phasespace and derivative-coupling factors. The three-photon decay of the $Z$ constitutes the best hope for a specific monopole-induced signature.

\section{A Digression ON MONOPOLIUM}

Given the strength of the coupling of monopoles to $Z$ 's and $\gamma$ 's (and of dyons to $W$ 's), monopolia, tightly bound states of a monopole and its antiparticle, should exist. Are they the signatures to look for in $e^{+} e^{-}$annihilation? Do they affect our previous considerations? I argue that both questions ought to be answered negatively.

The monopolium bound state is not tractable by analogy with positronium, or even charmonium. With a "fine structure" constant $\alpha_{g}>1$, one expects the constituent monopoles to be tightly bound and highly non-relativistic.

Various authors [19] have computed monopolium masses by "softening" the potential with the introduction of a non-vanishing extension of the monopoles, in ways premonitory of the properties of topological monopoles. They obtain mass estimates for the ground-state monopolium some 200 times (!) lighter than the sum of masses of the constituents. While these results are to be taken cum grano salis, they convey the presumably correct feeling that monopolium states should appear, in $e^{+} e^{-}$annihilations, well below the "open monopole" threshold. Why are monopolia not the objects to hunt for?

Another calculation that cannot be confidently performed in analogy with positronium is that of the lifetime of a monopolium state against its decay into 
vector bosons. Recall that, for the $3 \gamma$ decay of the $\left(J^{P}=1^{-}\right)$orthopositronium ground state:

$$
\Gamma=\left(2 m_{e}\right) \times \frac{\left(\pi^{2}-9\right)}{9 \pi} \alpha_{e}^{6} .
$$

Upon substitution of $\alpha_{e}$ for $\alpha_{g}$ one obtains a forcible hint of a foretold conclusion: monopolia ought to have a width much larger than their mass. If so, they are not prominent peaks.

I expect the collective effect of wide monopolia to be describable at the "partonic" level by the monopole-antimonopole loops we have dealt with, in analogy with the QCD description of the energy-smeared cross section for $e^{+} e^{-}$annihilation into hadrons [20]. This completes the argument whereby monopolia ought to be irrelevant in practice.

\section{6. $W$-PAIR PRODUCTION IN $e^{+} e^{-}$COLLISIONS}

Dyons have a potential incidence on the $Z W^{+} W^{-}$and $\gamma W^{+} W^{-}$Triple Gauge-Boson Vertices (TGVs) to be thoroughly studied at LEP-II. To discuss these effects, it may be useful to revamp one's souvenir of some known properties of the Standard TGVs.

In the Standard Model, the tree-level TGVs are described by a Lagrangian

$$
\begin{gathered}
\mathcal{L}_{S M}=-i \frac{e}{s}\left[\kappa_{e} W_{3}^{\nu}\left(W_{\mu \nu}^{\dagger} W^{\mu}-W_{\mu \nu} W^{\dagger \mu}\right)+\kappa_{m} W_{3}^{\mu \nu} W_{\mu}^{\dagger} W_{\nu}\right] \\
W_{3}^{\mu} \equiv s A^{\mu}+c Z^{\mu}
\end{gathered}
$$

with $W_{3}^{\mu}$ the electrically-neutral isovector potential, and $W_{i}^{\mu \nu} \equiv \partial^{\mu} W_{i}^{\nu}-\partial^{\nu} W_{i}^{\mu}$. In Eq. (24), $\kappa_{e}=\kappa_{m}=1$ are the non-anomalous "electric" and "magnetic" couplings of $W$ 's to $\gamma$ and $Z$. Radiative effects modify the coefficients $\kappa_{e}, \kappa_{m}$ of the (dimension $d=4$ ) couplings of $\mathcal{L}_{S M}$; the dispersive parts of these corrections are akin to the charge form factor and magnetic anomaly $(g-2)$ of an elementary fermion. 
Also in analogy with $g-2$, vertex corrections generate TGVs that are describable by effective interactions not appearing in $\mathcal{L}_{S M}$. The lowest dimension $(d=6)$ of these TGVs is

$$
\mathcal{O}_{W} \equiv W_{3}^{\mu \nu} W_{\nu \rho}^{\dagger} W_{\mu}^{\rho}
$$

The corresponding effective Lagrangian is $\mathcal{L}_{W}=a \mathcal{O}_{W}$, with $a$ of order $e \alpha /\left(\pi M^{2}\right)$ and $M$ representative of the mass of virtual particles. Effective operators such as $\mathcal{O}_{W}$ are particularly useful in discussing physics $[21,22]$ beyond the Standard Model.

As an example of non-standard effect, take a vector-like doublet of conventional leptons $\left(E^{0}, E^{-}\right)_{L, R}$ of degenerate mass $M>M_{Z} / 2$. This appendage affects the couplings of Eq. (24), entailing modifications that (after renormalization) are of the form $\Delta \kappa_{e}, \Delta \kappa_{m} \propto e^{3} q^{2} / M^{2}$. The vertex correction illustrated in Fig. 5a also induces effects that, to leading order in $1 / M$, are described by the addition to $\mathcal{L}_{S M}$ of the effective interaction:

$$
\mathcal{L}_{E}=-\frac{i}{240 \pi^{2} M^{2}}\left(\frac{e}{s}\right)^{3} \mathcal{O}_{W}
$$

Non-standard radiative effects may also induce CP-violating TGVs not included in $\mathcal{L}_{S M}$, two of which, of dimension $d=6$, have been discussed in the literature ${ }^{\star}, \widetilde{\mathcal{O}}_{\kappa} \equiv \widetilde{W}_{3}^{\mu \nu} W_{\mu}^{\dagger} W_{\nu}$, the dual sibling of the Standard "magnetic" coupling of Eq. (24), and

$\widetilde{\mathcal{O}}_{W} \equiv \widetilde{W}_{3}^{\mu \nu} W_{\nu \rho}^{\dagger} W_{\mu}^{\rho}$, the dual partner of $\mathcal{O}_{W}$. A third possibility, a dual counterpart of the "electric" $\left(\kappa_{e}\right)$ coupling of Eq. (24), has not been discussed; and this for a good reason: its coefficient at $q^{2}=0$ would be the magnetic-monopole charge of the $W$.

* The low-energy limits of couplings of the form $\widetilde{\mathcal{O}}_{\kappa}$ and $\widetilde{\mathcal{O}}_{W}$ describe electric dipole and magnetic quadrupole moments of the $W$ 's, and their electroweak generalizations. Their standard values are of very high order of perturbation theory, and negligible in practice. 
Consider, at long last, the $O\left(g e^{2}\right)$ vertex corrections of Figs. 5b,c, induced by our hypothetical dyon doublet $\left(D^{0}, D^{-}\right)_{L, R}$. We are interested in the lowestdimension TGVs characterizing this radiative effect or, more precisely, in the interference between the standard $e^{+} e^{-} \rightarrow W^{+} W^{-}$amplitude and the amplitude induced by this correction. For mass-degenerate dyons, the amplitudes of Figs. 5b,c add to zero, since $D^{-}$and $\bar{D}^{0}$ have opposite magnetic charges (the "Furry theorem" stating the vanishing of this TGV is in fact a consequence of the magnetic analogue of charge-conjugation symmetry, and applies to all orders of perturbation theory). To explore a possible non-vanishing effect, we momentarily lift our restriction to mass-degenerate dyons.

We saw in Section 2 that the dyon Feynman rules are not without blemish and, indeed, their use to compute the amplitudes of Figs. 5b,c leads to troubles identical to the ones we discussed there. To get out of this cul de sac, I simply conjecture that a consistent set of computational rules for dyons would result in an effective interaction analogous to the unequal-mass generalization of Eq. (27) but for the trading of $\mathcal{O}_{W}$ for its dual, $\widetilde{\mathcal{O}}_{W}$, and the substitutions of electric for magnetic coupling constants. In support of this conjecture, recall that there is no difficulty in "taking the dual" of an interaction involving $\vec{E}$ and $\vec{B}$ fields rather than the vector potentials, essentially all we suggest is to make the replacement $W_{\mu \nu} \rightarrow \widetilde{W}_{\mu \nu}$ in Eqs. (26),(27) while substituting leptons for dyons ${ }^{\dagger}$.

Let the split dyon masses be $m_{-}^{2}=m^{2}+\mu^{2}, m_{0}^{2}=m^{2}-\mu^{2}$. Following our conjecture and recalling that $e g=2 \pi n$, we may compute the dyon-induced TGV, for small $\mu^{2} / m^{2}$, as:

$$
\mathcal{L}_{D} \simeq \frac{i e n}{720 \pi c s^{2}} \frac{\mu^{2}}{m^{4}} \widetilde{B}^{\mu \nu} W_{\nu \rho}^{\dagger} W_{\mu}^{\rho},
$$

with $B^{\mu \nu}$ the derivative fields of the $U(1)_{Y}$ potential of Eq. (3). Behold! the operator in Eq. (28) is not of the $\epsilon^{i j k} W_{i} W_{j} W_{k}$ form of Eqs. (24),(26); sacrosanct on

$\dagger$ Dyons also induce effects proportional to $\widetilde{\mathcal{O}}_{\kappa}$, and a non-vanishing "magnetic-charge radius" of the $W$. We do not dwell on these effects, which are of the same order of magnitude as the ones we do discuss, and do not modify the conclusions. 
grounds of the gauge symmetry. The reason is that this operator is of dimension $d=8$, in spite of its $d=6$ disguise. The mass $m$, common to both (vector-like) dyons can be directly ascribed to an invariant term in the Lagrangian. The mass $\mu$, which splits them, must be proportional to a term such as $\bar{D} M^{0}\langle\Phi\rangle$, and the Higgs field $\Phi$ accounts for the extra field-dimensions.

The observability at LEP-II of " $T$-odd" effects ${ }^{\ddagger}$ induced by a CP-violating interaction such as Eq. (28) has been carefully investigated [23]. Even for the most optimistic case ( $\mu$ comparable to $m, m \sim 1 \mathrm{TeV}$ ) the coefficient in Eq. (28) is three orders of magnitude below the level required to observe a $1 \sigma$ effect in the combined results of four LEP-II experiments, each stockpiling some $10^{4} \mathrm{~W}$ pairs [23]. And, to make matters worse, the lower limits on the mass of non-degenerate dyons are much stronger than the limits we discussed in Section 3, for the same reason (a breaking of the "custodial" $S U(2)$ symmetry) that a split $(t, b)$ quark pair contributes so significantly to radiative corrections.

The moral of this Section is that there appears to be no hope for LEP-II, concerning monopoles and/or dyons, to improve on the exclusion or discovery capabilities of LEP-I. This is an example of a general result regarding the measurement of triple gauge-boson vertices [22]. I have not explored the potential of higher-energy $e^{+} e^{-}$colliders.

\section{Monopoles that aRe not Point-Like}

The very heavy composite monopoles that arise in Grand Unified Theories are certainly impossible to produce in current terrestrial laboratories. It is conceivable, however, that much lighter yet non-point-like monopoles exist, either for reasons that we have not at all fathomed, or because something like a Grand Unification actually takes place at an unsuspectedly low energy. It is therefore interesting to ponder what the low-energy signatures of composite monopoles might be.

$\ddagger$ An observable is $T$-odd if it changes sign as the spin and momenta of all particles are reversed. 
The grand-unified monopoles are classical or semi-classical solutions to a gauge theory. They do not correspond to a field appearing in the Lagrangian and, strictly speaking, they do not exist as virtual particles to be included in a quantum loop expansion, wherein only gauge bosons and other point-like particles should play a role. Analogously, only quarks and gluons, and perhaps their condensates, but not hadrons, ought to be used in pure-QCD perturbation theory (though useful alternative schemes do exist, such as the chiral Lagrangians).

Recall the foregone times when the quark model and QCD were not established. Imagine that the anomalous magnetic moment of muons and electrons had been measured, by then, to a sufficient precision for the hadronic contribution to the photon's vacuum polarization, $\Pi\left(q^{2}\right)$, to play a significant role. To have theory agree with experiment one would need to estimate this contribution. Use of a dispersion relation for $\Pi\left(q^{2}\right)$, and of $\pi^{+} \pi^{-}$intermediate states with a form factor, $F_{\pi}\left(q^{2}\right)$, describing the $\rho$ resonance, would result in an answer of the correct magnitude, even though one would have violated the dictum of the last paragraph: thou shalt not use but fundamental particles in a quantum correction ${ }^{\S}$.

For the rest of this Section, I imitate the procedure of the previous paragraph, trading pions for extended monopoles. I also trade a pion-like form factor for a proton-like one, since the monopole threshold should be above the monopolium states, like the $p \bar{p}$ threshold is above the $\rho$ mass. For the conventionally defined electric and magnetic form factors at threshold $\left(q^{2}=4 m^{2}\right)$ I set a normalization $G_{E}=G_{M} \sim 0.5$, the measured value for protons. Above threshold I let the form factors decrease as in a dipole fit, with a slope that corresponds to a mean square radius $\left\langle r^{2}\right\rangle=(\bar{\alpha} m)^{-2}$, with $\bar{\alpha}=1 / 40$.

With the use of conventional dispersion-relation techniques, one can redo our previous calculations of monopole-mass limits and the $Z \rightarrow 3 \gamma$ width. The mass limits of Eqs. (11)-(13) are weakened by a factor $\sim 3$, and the $3 \gamma$ branching ratio of Fig. 4 now reaches the interesting level of $1 \mathrm{ppm}$ also for monopoles that are

\footnotetext{
$\S$ I am indebted to Andy Cohen for insisting on this commandment, and on its exemptions.
} 
$\sim 3$ times lighter than the point-like ones. These masses and mass limits are very low, some $200 \mathrm{GeV}$. Consistency of the extended-monopole weak-coupling picture demands that the monopole-constituent gauge fields be much lighter than the monopole. For monopoles close to their lower mass limit, the novel gauge fields should already have been found!

The moral of this Section's bantam exercise is not unexpected. Not only are composite monopoles particularly difficult to produce, but it should be much easier to find their "constituents" than any of their indirect signatures.

\section{Cosmological Considerations}

Fossil remnants of any stable particle species may survive from the time when our Universe was hot enough to sustain a thermal population of its specimens, and monopoles are no exception. In a monopole-antimonopole symmetric Universe, conventional Big-Bang theory fixes the present relic monopole (and antimonopole) abundance in terms of the monopole mass and $\sigma \cdot v$, the velocity-weighed $M \bar{M}$ annihilation cross section $₫$. To an admittedly suspicious lowest order in $\alpha_{g}$, the total $\sigma \cdot v$ for annihilation into $Z Z, Z \gamma$ and $\gamma \gamma$ is:

$$
\sigma \cdot v \simeq \frac{4 \pi \alpha_{g}^{2}}{m^{2} c^{4}}
$$

where we have used $m \gg M_{Z}$, to be justified a posteriori. This result also applies to $D^{+} D^{-}$or $D^{0} \bar{D}^{0}$ (dyon) annihilation, since the additional annihilation of dyons into $W$-pairs proceeds with a conventional electroweak strength, and may be neglected.

Let $g$ and $g_{S M}$ be the number of degrees of freedom of a spin- $1 / 2$ monopole and of the complete Standard-Model zoo above the weak scale $\left(g=4, g_{S M}=427 / 4\right)$. Let $\Omega=\rho / \rho_{c}$ be the contribution of monopoles to the current universal energy density, in current critical units $\rho_{c} \sim 2 h^{2} 10^{-29} \mathrm{~g} / \mathrm{cm}^{3}$, with the Hubble-constant

ฯ An excessive abundance of topological monopoles was one of the original motivations for inflationary models [24]. We are exclusively dealing in this section with point-like monopoles and assuming inflation to have taken place at a temperature above the monopole mass. 
controversy still raging between $h \sim \frac{1}{2}$ and $h \sim 1$. For "cold relics" such as monopoles, a conventional calculation [25] results in the following estimates of the decoupling temperature $T_{d}$, and of $\Omega$ :

$$
\begin{gathered}
\frac{T_{d}}{m} \simeq\left[A-\frac{1}{2} \ln (A)\right]^{-1} \quad\left(A \equiv 0.038 g g_{S M}^{-1 / 2} M_{P} m \sigma \cdot v\right) \\
\Omega h^{2} \simeq\left(\frac{1.110^{12}}{1 \mathrm{TeV}}\right) \frac{m}{T_{d}} \frac{1}{\sqrt{g_{S M}} M_{P} \sigma \cdot v}
\end{gathered}
$$

with $M_{P}$ the Planck mass.

Equations (29)-(31) imply that, for monopoles or dyons to avert "overclosing" the Universe (contributing an $\Omega>1$ ), their mass must obey the restrictions:

$$
\begin{gathered}
m<8.7 n h \mathrm{PeV} \text { [monopoles] } \\
m<6.1 n h \mathrm{PeV} \text { [dyons]. }
\end{gathered}
$$

A primordial monopole-antimonopole asymmetry would make the mass limits more stringent. Recall Eqs. (11),(12) (and that $1 \mathrm{PeV}=10^{3} \mathrm{TeV}$ ) to conclude that there is room for point-like monopoles only in a mass interval of some four to five octaves.

\section{Conclusions}

Magnetic monopoles are some of the most interesting particles that we have not found.

The "official" non-elementary topological monopoles are too heavy to be made in the laboratory. Unsuspectedly light extensive monopoles are also difficult to produce, much as a nucleus of anti-helium, due to a severe "form-factor" suppression. Neither are their indirect signatures, as we have discussed, a good way to look for them. 
The virtual effects of point-like monopoles and dyons are observable in experiments conducted at energies below their production threshold. We have exploited the current data to set lower limits of the order of $1 / 2$ of a $\mathrm{TeV}$ on the masses of monopoles and degenerate dyons, and the standard Big-Bang cosmology to set upper limits of the order of $5000 \mathrm{TeV}$.

In the allowed mass interval, an unsuspectedly large $Z \rightarrow \gamma \gamma \gamma$ branching ratio would be the most specific currently accessible signature for the existence of magnetic poles.

Acknowledgements. I am indebted to Luis Alvarez-Gaume, Savas Dimopoulos, Belen Gavela, Howard Georgi, Gian Giudice, Shelly Glashow, Pilar Hernandez and Ryan Rohm for illuminating discussions, and I thank Andy Cohen for very many useful, constructive and instructive comments. I thank Elias Kiritsis, Tim Morris and Wolfgang Lerche for their exquisite patience with my computer illiteracy. I am indebted to Belen Gavela for a critical reading of the typescript and I thank Samuel Ting, and many other members of the L3 experiment, for discussions on the measurement of photons at LEP.

\section{REFERENCES}

1. P.A.M. Dirac, Proc. Roy. Soc. (London) Ser. A133 (1931) 60.

2. J. Schwinger, Phys. Rev. 151 (1966) 1048, 1055.

3. G. 't Hooft, Nucl. Phys. B79 (1974) 276;

A.M. Polyakov, JETP Lett. 20 (1974) 194.

4. E.B. Bogomolny, Soviet J. Nucl. Phys. 24 (1976) 801;

L.D. Fadeev, Lett. Math. Phys. 1 (1976) 289;

S. Coleman et al., Phys. Rev. D15 (1977) 544.

5. J. Schwinger, Phys. Rev. 144 (1966) 1087. 
6. B. Zumino, in Int. School of Physics, Ettore Majorana, edited by A. Zichichi (Academic, New York, 1966);

S. Weinberg, Phys. Rev. 138 (1965) B988;

J.C. Taylor, in Lectures in High Energy Physics, edited by H.H. Aly (Wiley, New York, 1968);

A. Rabl, Phys. Rev. 179 (1969) 1363.

7. D. Zwanziger, Phys. Rev. D3 (1971) 880, D6 (1972) 458.

8. W. Deans, Nucl. Phys. B197 (1982) 307.

9. E. Dagotto and J. Kogut, Nucl. Phys. B295 (1988) 123;

J. Kogut, E. Dagotto and A. Kočič, Phys. Rev. Lett. 60 (1988) 722;

S. Hands and R. Wensley, Phys. Rev. Lett. 63 (1989) 2169.

10. D.C. Kennedy and B.W. Lynn, Nucl. Phys. B322 (1989) 1;

D.C. Kennedy et al. Nucl. Phys. B231 (1989) 83;

B.W. Lynn, Stanford Univ. Preprint SU-ITP-867 (1989);

M.E. Peskin and T. Takeuchi, Phys. Rev. Lett. 65 (1990) 964.

11. H. Georgi, in Weak Interactions and Modern Particle Theory (Benjamin/Cummings Pubs. Menlo Park, 1984).

12. T.T. Wu and C.N. Yang, Nucl. Phys. B107 (1976) 365.

13. V.A. Novikov, L.B. Okun and M.I. Vysotsky, Nucl. Phys. B387 (1993) 35.

14. F. Abe et al., The CDF collaboration, Fermilab-PUB-94/097-E. May 1994.

15. S. Graf, A. Schafer and W. Greiner, Phys. Lett. B262 (1991) 463.

16. W. Heisenberg and H. Euler, Z. Phys. 98 (1936) 714, J. Schwinger, Phys. Rev. 82 (1951) 664, 93 (1954) 615, 94 (1954) 1362.

17. E.W.N. Glover and A.G. Morgan, Z. Phys. 60 (1993) 175.

18. F. Berends and R. Kleiss, Nucl. Phys. B186 (1981) 22. 
19. C.J. Goebel, in Quanta, Essays in Theoretical Physics, Dedicated to Georg Wentzel, edited by P.G.O. Freund, C.J. Goebel and Y. Nambu (Chicago, 1970);

E. Kyriakopoulos and R. Ramachandran, Nuovo Cim. Lett. 15 (1976) 161.

20. A. De Rujula and H. Georgi, Phys. Rev. D13 (1975) 1296;

E.C. Poggio, H.R. Quinn and S. Weinberg, Phys. Rev. D13 (1975) 1958.

21. A.C. Longhitano, Phys. Rev. D22 (1980) 1166 and Nucl. Phys. B188 (1981) 118 ;

T. Appelquist, in Gauge Theories and Experiments at High Energies, edited by K.C. Brower and D.G. Sutherland, (Scottish University Summer School in Physics Publ., St. Andrews 1980);

W. Buchmuller and D. Wyler, Nucl. Phys. B268 (1986) 621.

22. A. De Rujula, M.B. Gavela, P. Hernandez and E. Masso, Nucl. Phys. B384 (1992) 3.

23. A. Bilal, E. Masso and A. De Rujula, Nucl. Phys. B355 (1991) 549.

24. A.H. Guth, Phys. Rev. D23 (1981) 347;

A.D. Linde, Phys. Lett. 108B (1982) 389;

A. Albrecht and P.J. Steinhardt, Phys. Rev. Lett. 48 (1982) 1220.

25. For a review, see E.W. Kolb and M.S. Turner, The Early Universe (AddisonWesley Pub. Co. New York 1990). 


\section{FIGURE CAPTIONS}

1) (a) A one-loop virtual-monopole correction to the propagator of neutral vector bosons $V=Z, \gamma$. (b) A two-loop correction.

2) Lower limits on $m / n$ as a function of $m_{t}$, with $m$ the monopole mass and $n$ its charge in elementary units, (a) at the $1 \sigma$ confidence level, (b) at $3 \sigma$. The shaded areas are allowed. The recently published CDF result on $m_{t}$ narrows the allowed domain down to the barred regions.

3) $Z$-production in $e^{+} e^{-}$annihilation and monopole-induced $Z \rightarrow 3 \gamma$ decay; (a) with the dual photon vertices (denoted by a heavy dot) hooked to the monopole line and the standard vertex on the electron line. (b) A dual viceversa. (c) A higher-order correction to (a). (d) The QED background.

4) Branching ratio for monopole-induced $Z \rightarrow 3 \gamma$ decay, as a function of $m / n$, in the notation of Fig. 2.

5) Corrections to the $V W^{+} W^{-}$vertex, with $V=Z$, $\gamma$. (a) A lepton loop. (b,c) Two dyon loops. Only the heavy dots are magnetic couplings. 




[0 0 0 612 792]7273fg5.ps 